\title{
Factors affecting survival in 267 consecutive patients undergoing surgery for spinal metastasis from renal cell carcinoma
}

\author{
Clinical article
}

\author{
Claudio E. Tatsui, M.D., ${ }^{1}$ Dima Suki, Ph.D., ${ }^{1}$ Ganesh Rao, M.D., ${ }^{1}$ \\ Stefan S. Kim, M.D., ${ }^{2}$ AbhiJit Salaskar, M.D., ${ }^{1}$ Mustafa Aziz Hatiboglu, M.D., ${ }^{1}$ \\ Ziya L. Gokaslan, M.D., ${ }^{3}$ Ian E. McCutcheon, M.D., ${ }^{1}$ and Laurence D. Rhines, M.D. ${ }^{1}$ \\ ${ }^{1}$ Department of Neurosurgery, The University of Texas MD Anderson Cancer Center, Houston, Texas; \\ ${ }^{2}$ Department of Neurosurgery, Lahey Clinic Medical Center, Burlington, Massachusetts; and ${ }^{3}$ Department of \\ Neurosurgery, Johns Hopkins University School of Medicine, Baltimore, Maryland
}

\begin{abstract}
Object. Renal cell carcinoma (RCC) frequently metastasizes to the spine, and the prognosis can be quite variable. Surgical removal of the tumor with spinal reconstruction has been a mainstay of palliative treatment. The ability to predict prognosis is valuable when determining the role and magnitude of surgical intervention in cancer patients. To better identify factors affecting survival in patients undergoing surgery for spinal metastasis from RCC, the authors undertook a retrospective analysis of a large patient cohort at a tertiary care cancer center.

Methods. Relevant clinical data on a consecutive series of patients who had undergone surgery for spinal metastasis of RCC between 1993 and 2007 at The University of Texas MD Anderson Cancer Center were retrospectively reviewed. Demographic data, histopathological grade of primary tumor, timing of spinal surgery relative to diagnosis, treatment history prior to surgery, neurological status, and systemic disease burden were analyzed to determine the impact of these factors on survival outcome.

Results. The authors identified 267 patients who met the study criteria. Five-year overall survival (OS) after spine tumor resection was 7.8\%, with a median OS of 11.3 months (95\% CI 9.5-13.0 months). Patients with Fuhrman Grade 4 RCC had a median OS of 6.1 months (95\% CI 3.5-8.7 months), which was significantly lower than the 14.3 months (95\% CI 9.1-19.4 months) observed in patients with Fuhrman Grade 3 or less RCC ( $<<0.001)$. Patients with preoperative neurological deficits had a median survival of 5.9 months (95\% CI 4.1-7.7 months), which was significantly lower than the 13.5 months (95\% CI 10.4-16.6 months) observed in patients with a normal neurological examination $(\mathrm{p}<0.001)$. Patients whose spine was the only site of metastasis had a median OS of 19 months $(95 \%$ CI 9.8-28.2 months) after surgery, significantly longer than the 9.7 months (95\% CI 8.1-11.3 months) observed in patients with additional extraspinal metastasis sites $(\mathrm{p}<0.001)$. Patients with nonprogressing extraspinal metastasis (no metastasis, stable, or concurrent) had a median survival of 20.6 months (95\% CI 15.1-26.1 months), compared with 5.6 months (95\% CI 4.4-6.8 months) in patients with progressing metastasis $(\mathrm{p}<0.001)$.

Conclusions. The authors identified several factors influencing survival after spine surgery for metastatic spinal RCC, including grade of the original nephrectomy specimen, activity of the systemic disease, and neurological status at the time of surgery. These clinical features may help to identify patients who may benefit from aggressive surgical intervention.
\end{abstract}

(http://thejns.org/doi/abs/10.3171/2013.9.SPINE13158)

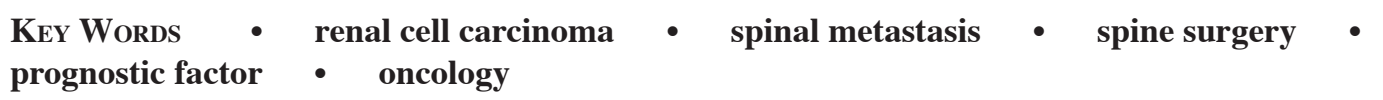

$\mathrm{R}$ ENAL cell carcinoma (RCC) is the most common primary malignancy of the kidney, with an estimated incidence of more than 50,000 new cases per year in the US ${ }^{23,24}$ Surgical removal of the primary tumor in the early stages of the disease is the potential cure,

Abbreviations used in this paper: $\mathrm{mTOR}=$ mammalian target of rapamycin; OS = overall survival; RCC = renal cell carcinoma. but unfortunately local recurrence or metastatic disease develops in many patients after initial treatment. ${ }^{6}$ Conventional immunotherapy with interferon or interleukin-2 has an estimated response rate of $10 \% .{ }^{30}$ New treatment modalities such as tyrosine kinase inhibitors, monoclonal antibodies, and mammalian target of rapamycin (mTOR) inhibitors have shown increased progression-free survival rates, ${ }^{7}$ but disease control is still an issue, ${ }^{19}$ and metastasectomy remains an important therapeutic modality, 


\section{Factors affecting survival after spine surgery for metastatic RCC}

frequently used to reduce the tumor burden and improve the chances of local control and survival. ${ }^{26,27}$

The skeleton is the second most common site of RCC metastasis, and approximately $40 \%$ of bony metastases occur in the spine. ${ }^{46}$ When RCC does spread to the spine, median survival is an estimated 10 months. ${ }^{31}$ Spinal metastases may also result in vertebral collapse and/or epidural spinal cord compression with a significant negative impact on quality of life and survival. ${ }^{21}$ Given the historically poor response of spinal metastases of RCC to standard systemic therapies and external beam radiotherapy, oncologists have had a low threshold for referring patients for surgical management. ${ }^{17}$ Even with recent advances in systemic treatment ${ }^{7}$ and radiation, ${ }^{14}$ patients with spinal instability or disease close to the spinal cord may be best treated with surgical intervention. ${ }^{32}$ Surgical series specifically analyzing RCC metastatic to the spine have reported a median survival ranging from 8 to 13 months. ${ }^{16,22,25,38}$ But these studies have failed to yield factors predicting survival, although long-term survivors have been documented. ${ }^{22}$

Our objective in the current study was to analyze a large number of patients undergoing spinal surgery for RCC metastases to identify factors associated with survival. Given the somewhat variable natural history of the disease, prognostic information would be especially valuable for counseling patients and assessing treatment strategies. Patients with a more favorable prognosis may warrant more aggressive intervention, while those with limited survival may be spared from surgeries whose benefit does not outweigh the morbidity. Moreover, such an analysis may reveal novel prognostic factors that are applicable to all patients being treated for spinal metastatic disease.

\section{Methods}

\section{Patient Population}

The study was reviewed and approved by the institutional review board. We examined data from all consecutive patients who had undergone surgery for spinal metastases from RCC at The University of Texas MD Anderson Cancer Center in the period from 1993 to 2007. Prospectively collected data in the Brain and Spine Database maintained at the institution were used for this study. A retrospective review of the hospital records and radiographic studies in patients was performed. We collected data on patient demographics, date of diagnosis of the primary kidney cancer, history and date of prior nephrectomy, histopathological grade of primary tumor including Fuhrman grade of the nephrectomy specimen (when available), timing of spinal surgery relative to diagnosis, date and location of first metastasis, date and location of the spinal metastasis, previous treatments (including chemo-, immuno-, and/or radiotherapy), neurological deficits prior to spine surgery, total number of spinal and extraspinal sites, and disease status (none, concurrent, stable, or progressing) of extraspinal metastases at the time of surgery. Date of death, when available, was confirmed using the Social Security Death Index.
Preoperative Evaluation and Classification of the Status of Systemic Disease

All patients in our series were monitored by personnel in the Genitourinary Oncology Department at the MD Anderson Cancer Center, where systemic radiological investigation was performed on a regular basis every 3-4 months to restage patients and evaluate treatment efficacy or the need for additional therapy. Imaging was also performed immediately prior to spine surgery to assess overall disease status. We reviewed all imaging studies obtained before the spine surgery, which included plain radiographs; bone scans; axial CTs of the chest, abdomen, and pelvis; MR images of the spine; and, when available, CTs and MR images of the brain. All patients underwent the previously described systemic workup before surgery, and the preoperative metastatic burden was noted (number and size, which were both considered significant for analysis). The extraspinal metastatic disease was classified as present or absent. If present, it was subclassified according to 1 of 3 sites: 1) lungs; 2) visceral organs other than the lungs, including the brain; and 3) bone other than the spine. Extraspinal disease was also categorized by status: 1) concurrent, evidence of metastatic disease only on the most recent radiographic investigation prior to spine surgery; 2) progressing, evidence of enlargement or increase in number on 2 consecutive radiographic investigations prior to spine surgery, and 3) stable, no change in size or number on 2 consecutive examinations prior to spine surgery. All patients had at least 1 spinal metastasis prompting the referral for spine surgery.

\section{Data Analysis}

Frequencies and descriptive statistics of demographic and clinical variables were calculated. Data were analyzed using SPSS version 12.0.1 (SPSS Inc.). The KaplanMeier method was used to estimate postoperative survival, and survival curves were compared using a log-rank test. The Cox proportional hazards model was used to identify factors associated with survival after spine surgery at the univariate and multivariate levels. Rate ratios and their corresponding $95 \%$ confidence intervals were computed. A time-dependent covariate analysis was performed to assess the effect of a post-spine surgery metastasis on postoperative duration of survival. The Stata software (Release 10) was used for this analysis. A probability value $\leq 0.05$ was considered statistically significant.

\section{Results}

Retrospective review of our database and patient charts provided all preoperative data fields and the length of survival used in our analysis. No individual was excluded. Two hundred sixty-seven patients with RCC metastatic to the spine underwent initial surgical treatment and were included in our analysis. There were 206 males (77\%) and 61 females (23\%). The median patient age at the time of spinal metastasis diagnosis was 58 years (range 15-86 years), and the median age at the time of surgery was 59.2 years (range 17-86 years). Experienced surgeons performed all procedures at a tertiary care can- 
cer center, and the surgical technique consisted of intralesional excision, typically to remove the maximum gross disease, with decompression of the spinal cord, followed by reconstruction of the affected vertebral bodies and stabilization of the spine. When feasible, preoperative embolization was performed within 36 hours before the procedure with the intent to reduce intraoperative blood loss. The median follow-up for all surviving patients was 77.9 months (range 28-158 months).

\section{Survival After Spine Surgery}

The survival rates after spine surgery at $1,2,3$, and 5 years was $47 \%, 30 \%, 20 \%$, and $8 \%$, respectively. The median overall survival (OS) after spine surgery was 11.3 months (95\% CI 9.5-13.0 months). The variables that did not have a significant univariate effect on OS after spine surgery were age, sex, latency between the primary and metastatic disease, latency between the primary disease and spinal metastasis, prior nephrectomy, previous radiation to the spine, and postoperative complications.

\section{Nephrectomy Prior to Spine Surgery and Fuhrman Nuclear Grade}

Overall, surgical removal of the primary kidney cancer before surgery for the metastatic spinal disease did not influence survival after the latter procedure (data not shown). Nuclear grading of the nephrectomy specimen according to the Fuhrman classification ${ }^{13}$ was significantly associated with survival after spine surgery. Patients with a Fuhrman Grade 4 tumor had a median survival of 6.1 months (95\% CI 3.5-8.7 months) after spine surgery, which was significantly lower than the 14.3 months $(95 \%$ CI 9.1-19.4 months) seen when the Fuhrman grade was 3 or less ( $\mathrm{p}<0.001$, log-rank test), with a univariate HR of 2.4 (95\% CI 1.6-3.5, p < 0.001, Cox proportional hazards model; Fig. 1A).

\section{Adjuvant Systemic Therapy Prior to Spine Surgery}

One hundred eighteen patients (44\%) had prior immuno- or chemotherapy before the spine surgery. The median survival for these patients after spine surgery was 8.5 months (95\% CI 6.5-10.6 months), significantly lower than the 13 months (95\% CI 10.0-15.8 months) observed in the 149 patients $(56 \%)$ who had the spine surgery before any systemic treatments $(p=0.009$, log-rank test).

\section{Radiation Therapy Prior to Spine Surgery}

Radiation therapy was administered to 99 patients $(37 \%)$ as initial treatment for the spinal metastasis. The median interval between radiation and surgical treatment was 4 months (range $0.2-46$ months). The median survival of patients who had prior radiation therapy for the spine disease was 10.8 months (95\% CI 7.6-13.9 months), which was not statistically different from the 11.1 months $(95 \%$ CI 8.9-13.2 months) when surgery was the first local treatment for the spinal metastasis ( $\mathrm{p}=0.49$, log-rank test). The complication rate after surgery was not influenced by history of radiation therapy. We observed complications in 23 (14\%) of 168 patients who did not have radiation prior to the spine surgery and in $12(12 \%)$ of 99 patients who did.

\section{Neurological Status Prior to Spine Surgery}

Preoperative neurological deficits (Frankel grade < E) were present in 50 patients (19\%). These patients had a median survival of 5.9 months (95\% CI 4.1-7.7 months) after spine surgery, which was significantly shorter than the 13.5 months in the remaining 217 cases $(81 \%)$ with normal neurological examinations (95\% CI 10.4-16.6 months, $\mathrm{p}<0.001$, log-rank test). For this variable, a univariate HR of 2.3 (95\% CI 1.6-3.1, p < 0.001, Cox proportional hazards model) was observed (Fig. 1B).

\section{Extent of Spinal Metastatic Disease}

The extent of spinal metastatic disease at the time of the spine surgery was measured according to the regions involved and the number of vertebral levels affected. The thoracic spine was the most common location of spinal metastasis. We observed thoracic metastasis in 177 patients $(66 \%)$. The lumbar spine was the second most affected site in 88 patients (33\%); followed by the cervical spine in $29(11 \%)$ and the sacrum in 19 patients $(7 \%)$. Patients with disease in the cervical spine had a median survival of 7 months (95\% CI 4.5-9.5 months), which was significantly lower than the survival of patients with disease elsewhere, who had a median survival of 11.7 months (95\% CI 9.8-13.7 months, $\mathrm{p}<0.02$, log-rank test). The total number of spinal sites (contiguous vertebral levels treated as 1 site) affected by metastatic disease was a factor that influenced survival after spinal surgery only when there were 4 or more distinct sites of spinal disease. In the 11 patients in whom this observation was made, the median survival was 4.4 months (95\% CI $0.0-14.2$ months), which was lower than the 11.3 months (95\% CI 9.4-13.1 months) in the 256 patients who had 3 or fewer affected spinal sites ( $\mathrm{p}=0.05, \log$-rank test).

\section{Metastatic Disease Restricted to the Spine at the Time of Spine Surgery}

The spine was the only site of metastasis at the time of surgery in 70 patients (26\%), and the median survival for this group was 19 months (95\% CI 9.8-28.2 months). Of note, there were 9 patients with an extraspinal metastasis that had been resected without recurrence prior to spine surgery; these patients were considered to have an extraspinal site that was stable. The 197 patients (74\%) with evidence of extraspinal disease at the time of surgery had a median survival of 9.7 months (95\% CI 8.1-11.3 months), which was significantly shorter than survival in the group with metastatic disease restricted to the spine ( $p=0.002$, log-rank test). The univariate HR observed for metastatic disease restricted to the spine was 0.6 (95\% CI $0.5-0.8, p=0.001$, Cox proportional hazards model). The Kaplan-Meier estimate of survival is depicted in Fig. 1C.

\section{Latency of Systemic Metastasis After Spine Surgery for Patients With Solitary Spinal Metastasis}

Of the 70 patients with disease only in the spine, 44 had an isolated, single site (solitary metastasis) at the time of spine surgery. Their median survival of 23.9 months was significantly longer than the 11.5 months observed in the remaining 26 patients with 2 or more spinal disease 
Factors affecting survival after spine surgery for metastatic RCC
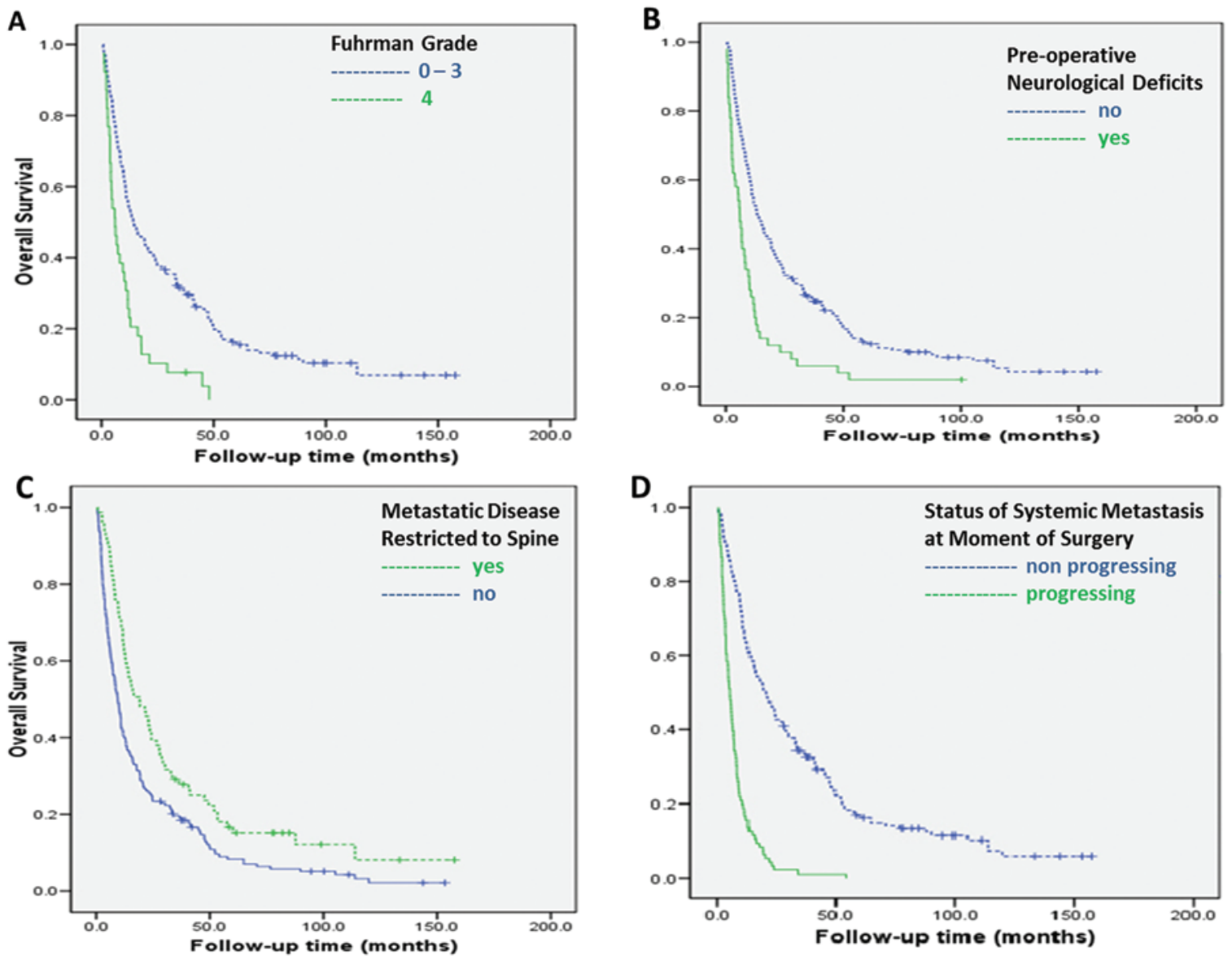

FIG. 1. Kaplan-Meier estimates of survival after spine surgery according to Fuhrman grade of the nephrectomy specimen (A), neurological status (B), presence of extraspinal disease (C), and progression of systemic disease (D).

sites ( $\mathrm{p}=0.007, \log$-rank test). The group of 44 patients was evaluated to determine if additional metastasis had appeared after spine surgery, which occurred (extraspinal or separate spinal site) in 33 individuals (75\%). The median time for the additional metastasis to develop was 12.2 months (95\% CI 2.1-22.3 months). The Kaplan-Meier estimates of the proportions having another metastasis were $14 \%$ at 3 months, $34 \%$ at 6 months, and $48 \%$ by 1 year after surgery. The Kaplan-Meier estimate of the proportion having an additional metastasis by the last follow-up was $82 \%$. In a time-dependent covariate analysis, the presence of an additional metastasis after spine surgery was associated with a decreased postoperative duration of survival (HR 4.2, 95\% CI 1.8-9.8, $\mathrm{p}=0.001$, Cox proportional hazards model). Of the 44 patients, 11 (25\%) died within 1 year of the spine surgery.

\section{Number of Extraspinal Sites of Metastasis at the Time of Spinal Surgery}

The most common location of metastases (in addition to the spine) was the lungs (124 patients [46\%]), followed by bone other than the spine (119 patients [45\%]), and visceral organs other than the lung and including the brain (75 patients [28\%]).

Based on these 3 groupings, the total number of extraspinal metastatic sites $(0,1,2$, or 3$)$ at the time of spine surgery was a strong predictor of survival. Of note, there were 9 patients with an extraspinal metastasis that had been resected without recurrence prior to the spine surgery. These patients were considered to have an extraspinal site that was stable. In the 70 patients with disease restricted to the spine (no extraspinal site), the median survival of 19 months (95\% CI 9.8-28.2 months) was significantly longer than the 8.4 months (95\% CI 5.7-11.1 months) observed in the 77 patients (29\%) with a spine metastasis plus 2 extraspinal sites, and the 4.8 months (95\% CI 2.5-7.1 months) in the 23 patients (9\%) with a spine metastasis plus 3 extraspinal sites ( $p<0.001$, logrank test). The location of the extraspinal metastasis did not influence survival after spine surgery.

\section{Status of the Systemic Disease at the Time of Spine Surgery}

Seventy patients (26\%) had no evidence of extraspinal metastasis, $38(14 \%)$ had stable systemic disease, and 63 (24\%) had concurrent extraspinal metastasis (discovered at the time of spinal surgery and therefore growth status could not be determined). The median survival of 19.0 months (95\% CI 9.8-28.2 months) after spine surgery in the 70 patients without extraspinal metastasis was not significantly different from the 33.4 months $(95 \%$ CI 22.6-44.3) observed in the 38 patients with stable extraspinal disease ( $\mathrm{p}=0.3, \log$-rank test) or the 16.2 months 


\section{E. Tatsui et al.}

(95\% CI 7.2-25.1) seen in the 63 patients with concurrent extraspinal metastasis ( $\mathrm{p}=0.42, \log$-rank test). Therefore, to simplify our analysis, we combined these 171 patients into a group with "nonprogressing" extraspinal disease. This group had a median survival of 20.6 months $(95 \%$ CI 15.1-26.1 months).

Progressive extraspinal metastasis was observed in the remaining 96 patients $(36 \%)$ at the time of spine surgery. The median survival after spine surgery in these patients was 5.6 months (95\% CI 4.4-6.8 months) and was significantly lower than the 20.6 months observed in the nonprogressing group. A univariate HR of 4.1 (95\% CI 3.1-5.4, $\mathrm{p}<0.001, \log$-rank test) was observed for the status of systemic metastasis at spine surgery (Fig. 1D).

\section{Perioperative Complications and Recurrence Rate}

Complications occurred in 35 (13\%) of the 267 patients. In order of frequency, they were wound-related complications (11), pneumonia and sepsis (7), CSF leak (4), hardware failure (4), postoperative cauda equina syndrome (3), Ogilvie syndrome (1), neurogenic bladder (1), paraplegia (1), stroke (1), myocardial infarction (1), and epidural hematoma (1). The occurrence of complications did not affect survival after spine surgery. The 30-day mortality rate was $3 \%$ (9 cases total, 3 of which were directly attributable to the spine surgery). Patients who experienced a postoperative complication had a median survival of 10.2 months (95\% CI 4.5-16.0 months), compared with 11.7 months (95\% CI 9.8-13.6) in the 232 patients who did not have an adverse event related to spine surgery $(p=0.41, \log$-rank test).

The RCC recurred after the initial surgical treatment in 41 cases $(15 \%)$, and 36 of these cases had a second operation to decompress the spinal cord. The median survival of patients in these cases was 9.7 months.

\section{Multivariate Analysis}

The multivariate analysis revealed 3 significant variables associated with poor survival after spine surgery (Table 1). A Fuhrman Grade 4 (original) nephrectomy specimen was associated with an HR of 1.8 (95\% CI 1.2-2.6, $\mathrm{p}<0.003$, multivariate Cox proportional hazards model) as compared with RCC grades lower than 4. Patients with neurological deficits before spine surgery had an HR of 1.8 in the same model $(95 \%$ CI $1.2-2.7, \mathrm{p}<0.002)$ compared with the patients without deficits. But the factor most significantly associated with a poor survival outcome following spine surgery was radiographic progression of extraspinal metastasis prior to the spine surgery. This variable had an HR of 4.1 (95\% CI 2.9-5.8, p < 0.001).

TABLE 1: Cox multivariate regression: relative risk of death after spine surgery as relates to patient characteristics

\begin{tabular}{lll}
\hline \multicolumn{1}{c}{ Variable } & $\mathrm{HR}(95 \% \mathrm{Cl})$ & $\mathrm{p}$ Value \\
\hline Fuhrman grade (4 vs <4) & $1.8(1.2-2.6)$ & $<0.003$ \\
preop neurological deficit (yes vs no) & $1.8(1.2-2.7)$ & $<0.002$ \\
progressing systemic disease at & $4.1(2.9-5.8)$ & $<0.001$ \\
$\quad$ spine surgery (yes vs no) & & \\
\hline
\end{tabular}

\section{Discussion}

Patients with renal cell carcinoma (RCC) and metastatic disease have been reported to have a median survival of 10 months. ${ }^{31}$ However, the natural history of this condition can be quite variable, with some patients experiencing aggressive systemic progression and others presenting a more indolent course with long-term survival.

We focused our analysis on the outcome of 267 individuals who had received surgical treatment for metastatic RCC to the spine. The median OS after spine surgery was 11.3 months, consistent with previous reports in the literature ranging from 8 to 13 months. ${ }^{16,22,25,38}$ Our study is unique in terms of identifying several preoperative factors associated with improved survival after spine surgery (Table 1). The strongest factors included a nephrectomy specimen with a Fuhrman nuclear grade lower than 4, the absence of preoperative neurological deficits, and the lack of radiographic progression of extraspinal metastatic disease at the time of spine surgery.

Our analysis revealed interesting prognostic information not previously reported in the literature. Despite evidence that a nephrectomy followed by immunotherapy prolongs survival in patients with metastatic RCC, ${ }^{11,34}$ we were unable to demonstrate that removing the primary disease prior to the spine surgery improved survival in our sample, even in cases of isolated spinal metastasis. Therefore, we do not necessarily urge patients who present with a concurrent diagnosis of primary kidney cancer and spine metastasis to undergo a nephrectomy before or at the time of the spine surgery.

When a nephrectomy specimen was available, we found the Fuhrman nuclear grade ${ }^{13}$ to be a strong predictor of survival. In brief, this is a 4-tiered histological grading system based on nuclear morphology (nuclear size, nuclear shape, and nucleolar appearance) and assigned to the original nephrectomy specimen and is a well-established prognostic factor for the primary tumor. 9,10 The estimated annual growth rate of a metastatic lesion is higher than that for the primary disease, ${ }^{33}$ and there is a direct correlation between the Fuhrman nuclear grade of the primary tumor and the growth rate of systemic disease. ${ }^{5}$ To our knowledge, this report is the first to demonstrate the impact of Fuhrman grading on prognosis following surgery for spinal metastasis. Our multivariate analysis revealed that individuals with Fuhrman Grade 4 RCC had a significantly shorter survival than those with Grade 3 or lower, probably reflecting the more aggressive biological behavior of the underlying primary tumor.

The role of radiation therapy in the management of spinal metastasis is well established; 8,15 however, RCC is a relatively radioresistant histology, ${ }^{17}$ and the absence of local control with standard external beam radiation is reflected in our series by a median time of only 4 months to surgery after conventional radiation. Spinal stereotactic radiotherapy has emerged as a very promising treatment modality, and excellent control rates have been demonstrated in recent publications. ${ }^{14,32,45}$ But this treatment modality is not generally feasible in the setting of symptomatic spinal cord compression, ${ }^{14}$ and even with improved, high-dose, conformal radiation techniques, local control 
of RCC is poor when underdosed due to close proximity to the spinal cord ${ }^{4}$ Therefore, this technique is not a substitute for surgery but another weapon in the therapeutic armamentarium against spinal metastatic RCC. Most of our patients were treated before stereotactic spinal radiotherapy was widely available. It is unclear whether the use of this more conformal radiation modality in either the preoperative or postoperative setting will have any effect on the OS of these patients following surgery. Evaluation of its impact on surgical patients will need to be addressed in future studies.

Regarding adjuvant systemic therapy, we recognize that significant progress is ongoing with the development of targeted therapies, specifically tyrosine kinase inhibitors, monoclonal antibodies, and mTOR inhibitors. ${ }^{7}$ Today, the type and sequence of these treatments is highly variable from patient to patient. Most of our patients underwent surgery prior to the introduction of these new modalities, and since our study focuses on prognostic variables known at the time of surgery, we were unable to evaluate the impact of the postsurgical administration of these agents. Overall, we observed that patients who had undergone spine surgery before receiving chemo- or immunotherapy experienced a longer survival than those who had received these adjuvant therapies before spine surgery. The benefit of resection prior to biological therapy is documented in nodal and lung metastasis ${ }^{3}$ and could be inferred from our study. However, this observation may also reflect a selection bias, since patients in whom these regimens failed may have had more aggressive disease and fewer remaining treatment options after surgery.

Functional status is always a concern when dealing with spinal tumors, and the presence of neurological deficits predicts a poor prognosis in metastatic spinal disease. ${ }^{21,25,37}$ In their well-known surgical scoring system, Tokuhashi et al. listed the presence of neurological impairment as 1 of 6 critical factors affecting prognosis in patients undergoing surgery for spinal metastasis. ${ }^{40}$ Our findings clearly provided additional support for this notion, and we believe the best timing for a surgical intervention is prior to the development of neurological dysfunction. We observed in our multivariate analysis that median survival after surgery is significantly longer in patients with normal examinations than in those with compromised function (Fig. 1B).

Considering the biomechanical and functional implications associated with malignant disease affecting the spine, the presence of tumor in multiple spinal sites increases complexity and influences the extent of surgery. Interestingly, we found that multifocal disease in the spine was a negative prognostic factor only when 4 or more distinct sites (adjacent level considered a single site) were affected. Therefore, surgical treatment should not be precluded or limited based on the number of spinal foci, as even patients with 2 or 3 sites of spinal involvement could have reasonable survival. In terms of location, as we have noted for other histologies, ${ }^{1,36}$ the cervical spine was associated with a worse prognosis, perhaps because neurological dysfunction related to higher spinal cord levels is more severe than that related to caudal regions. Alternatively, cervical metastatic disease may be more difficult to eradicate because of the local anatomical complexity of this region.

In the context of systemic disease, the association between tumor burden and poor survival has been described. ${ }^{12,18,28,31}$ Series that focus on metastasis to nodes, ${ }^{3}$ lung, ${ }^{35}$ and bone ${ }^{2,29,43}$ have documented longer survival after surgical treatment of oligometastatic disease. This finding holds true in our analysis. We observed that individuals with metastatic disease restricted to the spine had significantly longer survival than patients with additional extraspinal disease (Fig. 1C). In particular, we examined a subgroup of 44 patients whose metastatic disease was limited to a solitary spinal site. The median survival after spine surgery (23.9 months) in this group was significantly better than in patients harboring multiple spinal sites regardless of their location. This result is consistent with other reports focusing on surgical management of a solitary metastasis from RCC. ${ }^{39,42}$ Given this longer survival, some authors have advocated en bloc resection, arguing that eradication of the single active site of disease may play a role in improving quality of life and even survival. 2,20,27,29,44 We offer what we believe is the first report providing information about the natural history of surgical patients presenting with a solitary metastasis of RCC to the spine. We found that $75 \%$ of these cases will develop an additional disease site (spinal or extraspinal) within a median interval of 12.2 months after the initial spine surgery. This observation may be used to support a more conservative initial approach in patients with isolated spinal metastasis, such as watchful observation, initiation of or change in chemotherapy regimen, or administration of stereotactic radiosurgery for the purpose of local control. Continued observation of these individuals may shed light on the natural history of the disease and allow more judicious use of aggressive surgery.

One of the most interesting aspects of our analysis relates to patients presenting with spinal metastasis in the context of extraspinal disease. In general, the location of the systemic disease is considered to have prognostic relevance since patients with metastases to the lungs tend to have better survival than patients with bony metastases. ${ }^{12}$ Our results are contrary to the assumption that an extraspinal disease location affects outcome given that we found no difference in survival after spinal surgery in patients with metastatic deposits in the lungs versus nonspinal bone versus visceral organs including the brain.

The extent of systemic involvement is one of the most important considerations in the management of patients harboring malignant neoplasia. Not surprisingly, it is a critical component of the 2 most widely used scoring systems predicting survival after surgical treatment of spinal metastasis. ${ }^{40,41}$ One hundred ninety-seven of our patients (74\%) presented with other extraspinal sites of metastasis at the time of spine surgery, and as predicted by those scoring systems, our univariate analysis confirmed that the number of sites (grouped as lung, visceral organs including brain, and bone other than spine) in addition to a spinal metastasis influenced survival (Table 2). Patients with $0,1,2$, or 3 sites of extraspinal metastasis had a median survival of $19.5,13.4,8.6$, and 4.5 months, respectively. Therefore, our data support the notion that patients 
TABLE 2: Univariate Cox regression: relative risk of death after spine surgery as relates to patient characteristics

\begin{tabular}{|c|c|c|}
\hline Variable & $\mathrm{HR}(95 \% \mathrm{Cl})$ & $\mathrm{p}$ Value \\
\hline Fuhrman grade $(4$ vs $<4)$ & $2.4(1.6-3.5)$ & $<0.001$ \\
\hline preop neurological deficits (yes vs no) & $2.3(1.6-3.1)$ & $<0.001$ \\
\hline cervical spine involved (yes vs no) & $1.6(1.1-2.4)$ & $<0.02$ \\
\hline $\begin{array}{l}\text { adjuvant therapy before spine surgery } \\
\text { (yes vs no) }\end{array}$ & $1.4(1.1-1.8)$ & 0.009 \\
\hline $\begin{array}{l}\text { total no. of spinal sites involved ( } \geq 4 \text { vs } \\
<4 \text { ) }\end{array}$ & $2.3(1.6-3.1)$ & 0.05 \\
\hline complications after surgery (yes vs no) & $1.2(0.8-1.7)$ & 0.41 \\
\hline $\begin{array}{l}\text { metastatic disease restricted to spine } \\
\text { (yes vs no) }\end{array}$ & $0.6(0.5-0.8)$ & 0.001 \\
\hline solitary spinal metastasis (yes vs no) & $0.6(0.4-0.8)$ & $<0.001$ \\
\hline $\begin{array}{l}\text { progressing systemic disease at time of } \\
\text { spine surgery (yes vs no) }\end{array}$ & $4.1(3.1-5.4)$ & $<0.001$ \\
\hline
\end{tabular}

with a significant systemic burden are less likely to benefit from extensive surgery.

We believe that knowledge of the biological activity of the systemic metastatic disease is an important piece of information when treating patients with spinal metastasis. The scoring system proposed by Tomita et $\mathrm{al} .^{41}$ recognizes "untreatable" visceral metastasis as a negative predic- tor of survival and is similar to the system developed by Tokuhashi et al.,.$^{40}$ which lists "nonremovable" involvement of visceral organs as a poor indicator of outcome. Unfortunately, these distinctions are somewhat subjective and may have nonuniform interpretation among surgeons and oncologists, especially between those practicing in specialized cancer centers and those working in smaller community hospitals. To our knowledge, a direct prognostic association between progressing metastatic lesions and survival after surgical treatment has not been reported, and this is a far more objective assessment. Patients with evidence of radiographic progression of extraspinal metastasis prior to spine surgery had a significantly lower median survival than patients with nonprogressing disease (Figs. 1D and 2). We believe that this information is critical since it allows objective identification of a subcategory of individuals with systemic disease that is expected to have a more aggressive course and faster progression and is associated with a decreased survival benefit following extensive surgical interventions aiming for local control of the spinal disease.

We identified several important prognostic variables; however, our study is subject to the weaknesses of a retrospective analysis, specifically the lack of a control or nonsurgical group for comparison. The intraoperative and perioperative events were not controlled or randomized; for example, not all patients underwent preoperative embolization and neither did the same surgeon perform

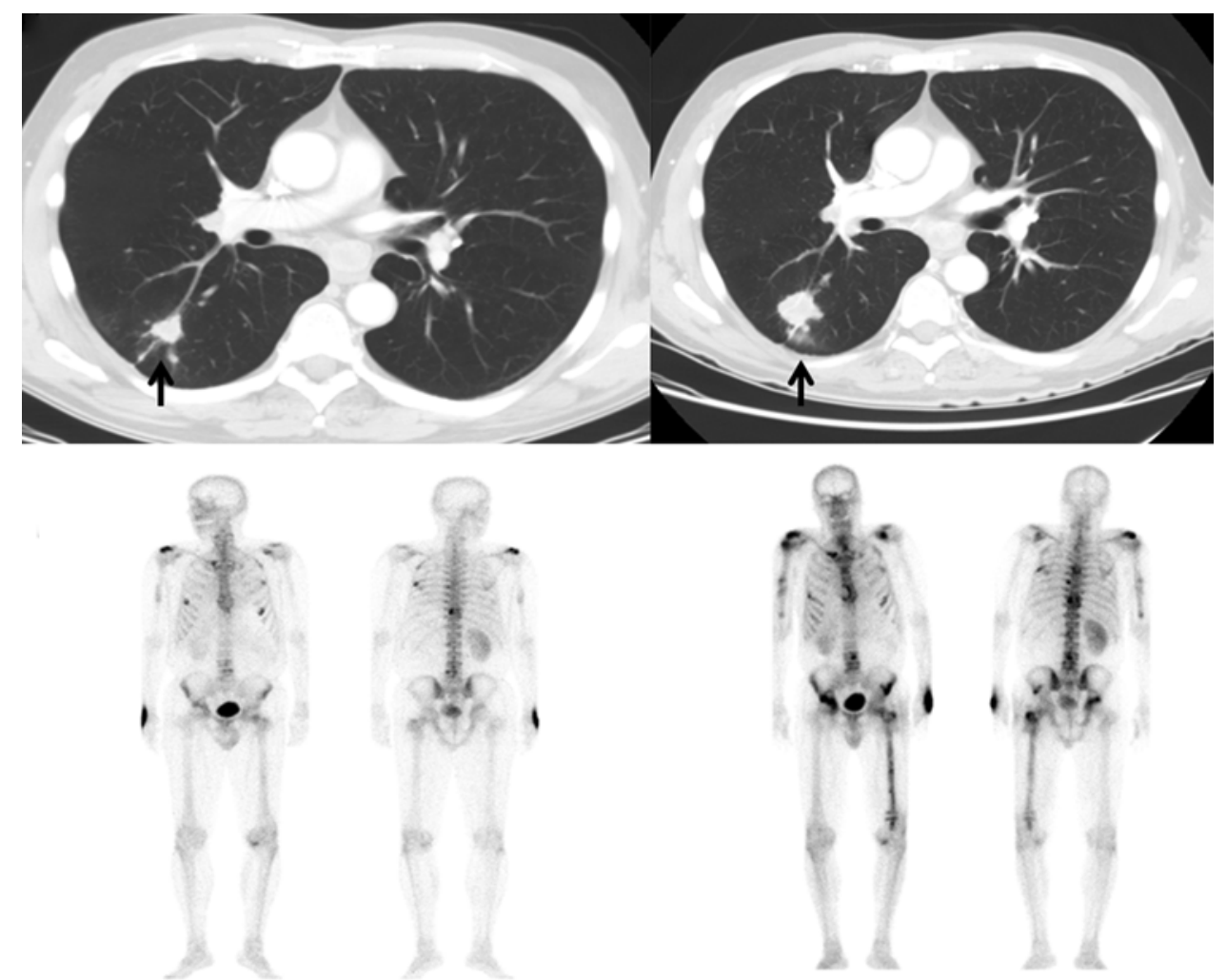

FIG. 2. Progression of metastatic disease. Upper: Consecutive CT scans of the chest obtained prior to spine surgery, demonstrating an increase in the size of a metastatic nodule on the posterior lobe of the right lung (arrows). These images were obtained in a patient who survived 4.8 months after spine surgery. Lower: Bone scintigrams demonstrating an increase in the number of metastatic lesions in 2 consecutive examinations before spine surgery. These images were obtained in a patient who survived 4.9 months after spine surgery. 


\section{Factors affecting survival after spine surgery for metastatic RCC}

all surgeries. Moreover, the postoperative systemic treatment was performed according to the preferences of the medical oncology teams. A prospective controlled study would be helpful in confirming our observations.

\section{Conclusions}

Despite the overall poor prognosis associated with metastatic RCC to the spine, there is individual variability in the biological behavior of this condition. We analyzed a large cohort of patients that underwent spine surgery for metastatic RCC and identified a series of factors affecting survival after surgical treatment. We report, for the first time, that a higher Fuhrman grade for the original nephrectomy specimen was a strong indication of the inherently aggressive biological behavior of the underlying disease. Patients with Fuhrman Grade 4 RCC had significantly worse survival after spine surgery than patients with Grade 3 or less RCC. As expected, preoperative functional status significantly affected survival after spine surgery, and patients with neurological dysfunction had a worse outcome than individuals without such impairment. Finally, we demonstrated that patients undergoing spine surgery for metastatic RCC restricted to the spine have better survival than those harboring extraspinal disease. We emphasize that knowing the extent and status of the systemic burden has prognostic significance, as radiographic progression of systemic disease before surgery had a strong negative effect on survival after surgery. Physicians treating patients with metastatic $\mathrm{RCC}$ to the spine should be aware of these variables and use this information to tailor the aggressiveness of the surgical treatment to optimize palliation and survival in these patients.

\section{Disclosure}

Dr. Rhines is a consultant for Stryker Spine. Dr. Gokaslan has direct stock ownership in Spinal Kinetics, US Spine, and AOSpine North America; and has received clinical or research support from AOSpine North America, Neurosurgery Research and Education Foundation, and DePuy for the study described.

Author contributions to the study and manuscript preparation include the following. Conception and design: Tatsui, Suki, Rao, Kim, Rhines. Acquisition of data: Tatsui, Rao, Kim, Hatiboglu, Gokaslan. Analysis and interpretation of data: Rhines, Tatsui, Suki, Rao, Salaskar, Kim, Hatiboglu, Gokaslan. Drafting the article: Rhines, Tatsui, Rao. Critically revising the article: all authors. Reviewed submitted version of manuscript: all authors. Approved the final version of the manuscript on behalf of all authors: Rhines. Statistical analysis: Suki, Salaskar. Study supervision: Suki.

\section{References}

1. Aizenberg MR, Fox BD, Suki D, McCutcheon IE, Rao G, Rhines LD: Surgical management of unknown primary tumors metastatic to the spine. Clinical article. J Neurosurg Spine 16:86-92, 2012

2. Althausen P, Althausen A, Jennings LC, Mankin HJ: Prognostic factors and surgical treatment of osseous metastases secondary to renal cell carcinoma. Cancer 80:1103-1109, 1997

3. Canfield SE, Kamat AM, Sánchez-Ortiz RF, Detry M, Swanson DA, Wood CG: Renal cell carcinoma with nodal metastases in the absence of distant metastatic disease (clinical stage
TxN1-2M0): the impact of aggressive surgical resection on patient outcome. J Urol 175:864-869, 2006

4. Chang EL, Shiu AS, Mendel E, Mathews LA, Mahajan A, Allen PK, et al: Phase I/II study of stereotactic body radiotherapy for spinal metastasis and its pattern of failure. J Neurosurg Spine 7:151-160, 2007

5. Crispen PL, Uzzo RG: The natural history of untreated renal masses. BJU Int 99 (5 Pt B):1203-1207, 2007

6. Eggener SE, Yossepowitch O, Pettus JA, Snyder ME, Motzer RJ, Russo P: Renal cell carcinoma recurrence after nephrectomy for localized disease: predicting survival from time of recurrence. J Clin Oncol 24:3101-3106, 2006

7. Escudier B, Szczylik C, Porta C, Gore M: Treatment selection in metastatic renal cell carcinoma: expert consensus. Nat Rev Clin Oncol 9:327-337, 2012

8. Faul CM, Flickinger JC: The use of radiation in the management of spinal metastases. J Neurooncol 23:149-161, 1995

9. Ficarra V, Martignoni G, Maffei N, Brunelli M, Novara G, Zanolla L, et al: Original and reviewed nuclear grading according to the Fuhrman system: a multivariate analysis of 388 patients with conventional renal cell carcinoma. Cancer 103:68-75, 2005

10. Ficarra V, Righetti R, Martignoni G, D’Amico A, Pilloni S, Rubilotta E, et al: Prognostic value of renal cell carcinoma nuclear grading: multivariate analysis of 333 cases. Urol Int 67:130-134, 2001

11. Flanigan RC, Salmon SE, Blumenstein BA, Bearman SI, Roy $\mathrm{V}$, McGrath PC, et al: Nephrectomy followed by interferon alfa-2b compared with interferon alfa-2b alone for metastatic renal-cell cancer. N Engl J Med 345:1655-1659, 2001

12. Frank W, Stuhldreher D, Saffrin R, Shott S, Guinan P: Stage IV renal cell carcinoma. J Urol 152:1998-1999, 1994

13. Fuhrman SA, Lasky LC, Limas C: Prognostic significance of morphologic parameters in renal cell carcinoma. Am J Surg Pathol 6:655-663, 1982

14. Gerszten PC, Burton SA, Ozhasoglu C, Vogel WJ, Welch WC, Baar J, et al: Stereotactic radiosurgery for spinal metastases from renal cell carcinoma. J Neurosurg Spine 3:288-295, 2005

15. Gerszten PC, Welch WC: Current surgical management of metastatic spinal disease. Oncology (Williston Park) 14:10131024, 1029-1030, 2000

16. Giehl JP, Kluba T: Metastatic spine disease in renal cell carcinoma-indication and results of surgery. Anticancer Res 19 (2C):1619-1623, 1999

17. Halperin EC, Harisiadis L: The role of radiation therapy in the management of metastatic renal cell carcinoma. Cancer 51: 614-617, 1983

18. Han KR, Pantuck AJ, Bui MH, Shvarts O, Freitas DG, Zisman A, et al: Number of metastatic sites rather than location dictates overall survival of patients with node-negative metastatic renal cell carcinoma. Urology 61:314-319, 2003

19. Heng DY, Xie W, Regan MM, Warren MA, Golshayan AR, Sahi C, et al: Prognostic factors for overall survival in patients with metastatic renal cell carcinoma treated with vascular endothelial growth factor-targeted agents: results from a large, multicenter study. J Clin Oncol 27:5794-5799, 2009

20. Hofmann HS, Neef H, Krohe K, Andreev P, Silber RE: Prognostic factors and survival after pulmonary resection of metastatic renal cell carcinoma. Eur Urol 48:77-82, 2005

21. Hosono N, Ueda T, Tamura D, Aoki Y, Yoshikawa H: Prognostic relevance of clinical symptoms in patients with spinal metastases. Clin Orthop Relat Res (436): 196-201, 2005

22. Jackson RJ, Loh SC, Gokaslan ZL: Metastatic renal cell carcinoma of the spine: surgical treatment and results. J Neurosurg 94 (1 Suppl):18-24, 2001 (Erratum in J Neurosurg 94 (2 Suppl):340, 2001)

23. Jemal A, Siegel R, Ward E, Hao Y, Xu J, Murray T, et al: Cancer statistics, 2008. CA Cancer J Clin 58:71-96, 2008 
24. Jemal A, Siegel R, Ward E, Murray T, Xu J, Thun MJ: Cancer statistics, 2007. CA Cancer J Clin 57:43-66, 2007

25. King GJ, Kostuik JP, McBroom RJ, Richardson W: Surgical management of metastatic renal carcinoma of the spine. Spine (Phila Pa 1976) 16:265-271, 1991

26. Kwak C, Park YH, Jeong CW, Lee SE, Ku JH: Metastasectomy without systemic therapy in metastatic renal cell carcinoma: comparison with conservative treatment. Urol Int 79: 145-151, 2007

27. Kwak C, Park YH, Jeong CW, Lee SE, Ku JH: No role of adjuvant systemic therapy after complete metastasectomy in metastatic renal cell carcinoma? Urol Oncol 25:310-316, 2007

28. Leibovich BC, Cheville JC, Lohse CM, Zincke H, Frank I, Kwon ED, et al: A scoring algorithm to predict survival for patients with metastatic clear cell renal cell carcinoma: a stratification tool for prospective clinical trials. J Urol 174:1759-1763, 2005

29. Lin PP, Mirza AN, Lewis VO, Cannon CP, Tu SM, Tannir NM, et al: Patient survival after surgery for osseous metastases from renal cell carcinoma. J Bone Joint Surg Am 89:17941801,2007

30. Motzer RJ, Bander NH, Nanus DM: Renal-cell carcinoma. N Engl J Med 335:865-875, 1996

31. Motzer RJ, Mazumdar M, Bacik J, Berg W, Amsterdam A, Ferrara J: Survival and prognostic stratification of 670 patients with advanced renal cell carcinoma. J Clin Oncol 17:2530-2540, 1999

32. Nguyen QN, Shiu AS, Rhines LD, Wang H, Allen PK, Wang XS, et al: Management of spinal metastases from renal cell carcinoma using stereotactic body radiotherapy. Int J Radiat Oncol Biol Phys 76:1185-1192, 2010

33. Oda T, Miyao N, Takahashi A, Yanase M, Masumori N, Itoh $\mathrm{N}$, et al: Growth rates of primary and metastatic lesions of renal cell carcinoma. Int J Urol 8:473-477, 2001

34. Pantuck AJ, Belldegrun AS, Figlin RA: Nephrectomy and interleukin-2 for metastatic renal-cell carcinoma. N Engl J Med 345:1711-1712, 2001 (Letter)

35. Pfannschmidt J, Hoffmann H, Muley T, Krysa S, Trainer C, Dienemann H: Prognostic factors for survival after pulmonary resection of metastatic renal cell carcinoma. Ann Thorac Surg 74:1653-1657, 2002

36. Sciubba DM, Gokaslan ZL, Suk I, Suki D, Maldaun MV, McCutcheon IE, et al: Positive and negative prognostic variables for patients undergoing spine surgery for metastatic breast disease. Eur Spine J 16:1659-1667, 2007

37. Sioutos PJ, Arbit E, Meshulam CF, Galicich JH: Spinal metas- tases from solid tumors. Analysis of factors affecting survival. Cancer 76:1453-1459, 1995

38. Sundaresan N, Scher H, DiGiacinto GV, Yagoda A, Whitmore W, Choi IS: Surgical treatment of spinal cord compression in kidney cancer. J Clin Oncol 4:1851-1856, 1986

39. Thyavihally YB, Mahantshetty U, Chamarajanagar RS, Raibhattanavar SG, Tongaonkar HB: Management of renal cell carcinoma with solitary metastasis. World J Surg Oncol 3:48, 2005

40. Tokuhashi Y, Matsuzaki H, Oda H, Oshima M, Ryu J: A revised scoring system for preoperative evaluation of metastatic spine tumor prognosis. Spine (Phila Pa 1976) 30:2186-2191, 2005

41. Tomita K, Kawahara N, Kobayashi T, Yoshida A, Murakami H, Akamaru T: Surgical strategy for spinal metastases. Spine (Phila Pa 1976) 26:298-306, 2001

42. Tongaonkar HB, Kulkarni JN, Kamat MR: Solitary metastases from renal cell carcinoma: a review. J Surg Oncol 49:4548, 1992

43. Toyoda Y, Shinohara N, Harabayashi T, Abe T, Akino T, Sazawa A, et al: Survival and prognostic classification of patients with metastatic renal cell carcinoma of bone. Eur Urol 52:163-168, 2007

44. Vogl UM, Zehetgruber H, Dominkus M, Hejna M, Zielinski CC, Haitel A, et al: Prognostic factors in metastatic renal cell carcinoma: metastasectomy as independent prognostic variable. Br J Cancer 95:691-698, 2006

45. Yamada Y, Bilsky MH, Lovelock DM, Venkatraman ES, Toner S, Johnson J, et al: High-dose, single-fraction imageguided intensity-modulated radiotherapy for metastatic spinal lesions. Int J Radiat Oncol Biol Phys 71:484-490, 2008

46. Zekri J, Ahmed N, Coleman RE, Hancock BW: The skeletal metastatic complications of renal cell carcinoma. Int J Oncol 19:379-382, 2001

Manuscript submitted March 5, 2013.

Accepted September 26, 2013.

Current affiliation for Dr. Hatiboglu: Department of Neurosurgery, Bezmialem Vakif University Medical School, Istanbul, Turkey.

Please include this information when citing this paper: published online November 8, 2013; DOI: 10.3171//2013.9.SPINE13158.

Address correspondence to: Laurence D. Rhines, M.D., 1515 Holcombe Blvd., Unit 442, Houston, TX 77030. email: lrhines@ mdanderson.org. 